\title{
Questions About Pesticides On Foods
}

Robert J. Geller, MD, FAAP

Associate Professor of Pediatrics, Emory University School of Medicine

\section{nemk}

American College of Medical Toxicology, www.acmt.net

REPRINTED FROM WWW.ACMT.NET

Funded by the Agency for Toxic Substances and Disease Registry Cooperative Agreement Number U61/ATU374416-01, Program Capacity to Conduct Environmental Medicine and Health Education Activities.

Produced in collaboration with the Association of Occupational and Environmental Clinics and the Pediatric Environmental Health Specialty Unit Program.

\section{SUMMARY}

Pesticides and other agriculturally applied chemicals often are present in food.

These chemicals have been shown to also be present in people in small amounts.

There are many ways that children are exposed to these chemicals. One way is through the food they eat. They are also exposed by the household and garden use of pesticides, living on or near farms where the chemicals are used, and by chemicals carried home on the clothes and skin of parents who work with and around pesticides.

The levels of exposure from food are rarely high enough to cause symptoms but they can affect the developing child, especially if exposed while in the womb or in infancy or early childhood.

1n The risks from the pesticides in foods are small compared to the tremendous health benefits of eating fruits and vegetables.

Agricultural chemicals are also found in animal products such as meat and dairy. Most of those chemicals are stored in the fat of the animal, so reducing the amount of animal fat in the diet will reduce your exposure to toxic chemicals and also reduce the other risks associated with excessive cholesterol and fat intake.

\section{Q. I've heard that foods are contaminated with pesticides. How does this happen?}

A. Modern agricultural practices are designed to produce as much good-tasting, appealing food as possible, and to minimize the amount of the crop that is spoiled by insects or other pests. These practices often include the use of chemicals, including pesticides used to protect food from pests, such as insects and rodents. Other chemicals also are used in the process of growing food, such as fertilizers used to enhance plant growth, herbicides to control weeds, fungicides to control mold and fungi, and antimicrobials to control bacteria. Pesticides can also be present in the livestock and fish's food and water. Different chemicals are applied at different times during the growing season, directed at the pests most likely to damage the crop at that time. The prudent use of pesticides reduces insect or other pest damage to the crops while they are growing. Proper agricultural practices can minimize the amount of pesticides present on the foods at the time of harvest.

Some pesticides enter the system of the plant, while others remain on the surface of the plant. Over a period of time, which varies from one crop to another, the systemic pesticides leave the plant. If the crop is harvested too soon after application of a systemic pesticide, a potentially dangerous amount of that pesticide may remain in the plant. 
The best choice for a farmer regarding which pesticide to use at a certain time varies, depending on the specific crop and the pests that need to be controlled at that point. Pesticides can often be avoided altogether by appropriate growing conditions (such as crop rotation practices) and use of less toxic alternatives. People growing fruits, vegetables, and grains intended for consumption should use pesticides only when needed, and only in the amount and timing recommended on the product label or by an Agricultural Extension Agent.

In summary, foods often contain some chemicals used in the growing process at the time they are sold for human consumption. The foods should contain as little of these added chemicals as possible and certainly less than the amount that causes effects on people.

\section{Q. I've heard that some pesticides found in foods are safer than others. Is this true?}

A. Yes, it is true. The toxicity of pesticides depends on several factors, including, the amount of time required for the chemical to break down to non-toxic materials (often measured in "half-lifes", the time required for half of the chemical to be broken down), how quickly it is eliminated from the body, whether or not it is metabolized (broken down) in the body, whether the products of metabolism are also toxic and whether or not it is used in combination with other pesticides that can have an additive effect. In general, the mechanism for toxicity is similar in both humans and insects, but much more pesticide is required to harm a person than an insect.

Several different types of insecticides and other pesticides are widely used in the United States. Outside the United States, other chemicals may be used in addition to those used in the U.S. All pesticides currently in use within the United States must be approved by the United States Environmental Protection Agency (called "registration") and then also by the Department of Agriculture in the state in which they are to be used. This registration process reviews the safety and effectiveness of the chemical, but each nonetheless poses risks if used inappropriately. The Food Quality Protection Act of 1996 requires that the EPA carefully evaluate children's exposure to pesticide residues in and on foods they most commonly eat, i.e., apples and apple juice, orange juice, potatoes, tomatoes, soybean oil, sugar, eggs, pork, chicken and beef. The EPA is also evaluating new and existing pesticides to ensure that they can be used with a reasonable certainty of no harm to adults as well as infants and children. This review also establishes the amount of each chemical that is allowed to be present in the food at the time it is sold in the U.S., considering the potential for some individuals to be vulnerable than others and trying to keep them safe as well.

The following pesticides are listed in order of relative frequency of use, not degree of toxicity.
- Organophosphate pesticides account for about one half of the insecticides used in the U.S. Organophosphate and carbamate insecticides (such as malathion, diazinon, guthion, azinphos, and chlorpyrifos) have been used extensively for more than 50 years. Excessive human exposure to these agents may result in clinically evident toxic effects. In addition, clinical effects may be difficult to separate distinguish from other illnesses.

Carbamates and organophosphates are cholinesterase inhibitors that kill insects by interfering with the proper functioning of their central nervous system. Cholinesterases are enzymes that are required to "turn off" signals once they are transmitted from one neuron to another within the nervous system. Preventing these enzymes from acting leaves the neuron that is receiving the message locked in the "on" position. Most animals have a reserve amount of cholinesterase activity, more than is needed to maintain normal function. Symptoms occur only when the amount of cholinesterase inhibition substantially exceeds the amount needed to maintain function. Insects are more sensitive to these agents than most people, which explains why insects can be killed while the people nearby are not adversely affected.

Cholinesterase inhibition can also occur in humans. The effects commonly noted after poisoning by these agents include a combination of nausea, vomiting, stomach cramps, diarrhea, excessive drooling and tearing, muscular weakness, and muscle tremors. Young children, however, may display sedation and other behavioral effects instead of these typical cholinesterase symptoms. Lingering effects have also been described, particularly after intense exposures.

- Carbamate compounds act similarly to organophosphates but the binding to acetylcholinesterase is reversible. Therefore, they have a shorter period of effect. Examples of carbamates include carbaryl, aldicarb, and propoxur. They are often less toxic to people than organophosphates, at similar doses.

- Pyrethroid compounds are less toxic to people than organo phosphates and carbamates. They are derived from chrysanthemums and are preferred for their rapid breakdown in the environment and low toxicity in humans. They occasionally produce allergic-like reactions in people and can exacerbate asthma. Examples of pyrethroids include pyrethroid, pyrethrin, resmethrin, and cypermethrin. These compounds affect the enzymes regulating insect wing motion, enzymes not found in humans.

Soap-based insecticides are used as an alternative to organo phosphates, carbamates, and pyrethroids. These products are often obtained from natural sources. These compounds have very low toxic potential to humans. 
- Long-acting anticoagulant rodenticides are used to kill rats, mice, and related pests. They act by preventing the blood of the animal from clotting after even minor injury, resulting in the pest bleeding to death. Because of the manner in which they are used in pest control, it is unlikely that they would get onto foods in sufficient quantity to pose a problem to humans or pets.

- Arsenic based pesticides have been used to control insects and rodents in the past. They are highly toxic to both animals and people, and are restricted to licensed pest control applicators in most U.S. jurisdictions. This class of compounds generally should not be used on products intended to be eaten.

- Zinc phosphide based pesticides have been used as fumigants to control insects and rodents in the past. They are highly toxic to both animals and people. Their chief advantage is their short life-span in the environment before decomposing. They are generally restricted to licensed pest control applicators. They leave little if any residue on the surface of foods treated with this agent.

In summary, the safest pesticides will have little effect on the person, do its intended job quickly and effectively, and then break down quickly in the environment. At the same time, this ideal amount that is absorbed into the human body would be broken down swiftly and efficiently into non-toxic compounds.

\section{Q. I've heard that some people are at higher risk from pesticides than others. Why?}

A. Variations in the ability of people to break down chemicals in their bodies occur because of genetic differences between individuals and changes that occur as people grow from infancy to adulthood. Individuals with low normal values before any exposure who are very young, or who take medications that alter cholinesterase activity, may be at higher risk from organophosphate or carbamate insecticides. For instance, among healthy people, the normal amount of cholinesterase enzyme activity varies greatly between individuals. If the average normal cholinesterase activity is defined as $100 \%$, then the normal range of activity is as large as $50 \%$ to $150 \%$ of the average value. The implication of this fact for people exposed to organophosphates or carbamates is that, where two people are exposed to the same amount of these pesticides, one can be extremely symptomatic and the other person can have no symptoms at all. Young infants may not yet have the enzymes to breakdown these compounds or may do so more slowly than adults. At the same time, children and infants may be exposed to much more pesticides per pound of body weight than adults in the same environment eating the same foods(see "I've heard that infants are at higher risk . . ." below for more details).

Some medications can slow down the metabolism of specific pesticides (particularly organophosphate insecticides). Medications that alter cholinesterase activity include, for example, some used to treat Alzheimer's Disease and related conditions (e.g., donepezil [Aricept ${ }^{\circledR}$ ], galantamine [Reminyl $®$ ], rivastigmine [Exelon $®]$, tacrine [Cognex $®]$ ), and some used to treat conditions such as myasthenia gravis (e.g., pyridostigmine $\left[\right.$ Mestinon $\left.{ }^{\circledR}\right]$ ). Also, individuals who have been exposed to these cholinesterase inhibitors, occupationally or from leisure activities (e.g., gardening) may be more susceptible upon further exposure.

In summary, some people are at increased risk of effects from pesticides. This may occur because of their genetic makeup, age, other exposures, illnesses, or other medications they use.

\section{Q. I've heard that infants are at higher risk than adults. Why?}

A. Infants and children are at a higher risk for exposure to pesticides and may be more susceptible to the toxic effects of these chemicals than adults.

Infants and children may be especially sensitive to health risks posed by pesticides for several reasons:

Their internal organs are still developing and maturing.

In relation to their body weight, infants and children eat and drink more than adults, possibly increasing their exposure to pesticides in food and water.

Certain behaviors-such as playing on floors or lawns or putting objects in their mouths-increase a child's exposure to pesticides used in homes and yards.

Pesticides may harm a developing child by blocking the absorption of important food nutrients necessary for normal healthy growth.

Infants have immature metabolic systems at birth, which gradually mature within the first few years of life. Both the infant's ability to metabolize (break down) various chemical compounds, and the infant's ability to eliminate them from their body through the urine, stool, or breathing may be reduced from that of an adult. This is because most of the enzymatic systems' maturation occurs within the first few months of life.

Infants also have a different exposure pattern to environmental chemicals than adults, for multiple reasons:

The diets of infants and children are different than adults diets. The child diet often is less varied. For example, toddlers eat disproportionately more apples, applesauce and apple juice and disproportionately more potato (much of it as "French Fries") than adults. This difference in diet results in chemical residues in apples and potatoes being eaten by children in relatively larger amounts; it is estimated to be as much as 16 times more potatoes and 4 times more apples than typical adults, based on the person's body weight. Children also drink more water than adults, as a component of infant 
formula and juice. For this reason, chemicals on or in water, apples, and potatoes may have a larger effect on infants and young children. Breast milk is a substantial water source for breast-fed infants, but the water is filtered by the mother's body which may or may not remove a specific chemical from the breast milk.

Infants spend more of their time close to the ground, crawling or lying on the floor. This is where compounds that are heavier than air concentrate and the child breathes them in. They also get exposed to dust (which may contain pesticide residues) settled on the floor, which children can place in their mouths through normal hand to mouth behaviors.

Infants breathe faster than adults. Normal infant respiratory rates are $20-40$ breaths per minute, while adults typically breathe $8-12$ times each minute. Infants are exposed to more air per minute per kilogram of body weight than adults, leading to the possibility of higher absorption of chemicals that they inhale.

Infants have a larger surface area of skin compared to their weight, leading to possibly greater intake of chemicals absorbed through the skin.

Also, for some chemicals, there are "critical periods" in fetal and infant human development when exposure to the chemicals can permanently alter the way an individual's biological system operates. The window of time during which this occurs varies, depending on the specific compound.

In summary, young infants are at potentially increased risk from pesticides and other agricultural chemicals because of their dietary habits (which may lead to relatively increased intake compared to adults), their metabolic immaturity, and their ongoing growth.

\section{Q. I've encountered conflicting opinions about the safety of certain chemicals from different experts. Why is there such disagreement?}

A. The data that is available to judge safety is limited. Much of it comes from animal studies, and it is difficult to determine exactly how to apply this information to people. Humans may be more or less sensitive than the species in which the tests were carried out, and certain effects occur only in specific species or only at certain stages of development in the womb. For example, thalidomide is a drug that causes incomplete development of arms when the human fetus is exposed at a specific stage of development, but this effect occurs in only a few species and was not observed in animal studies conducted before human use. Some fungicides, such as Cap$\tan { }^{\circledR}$, in current use are chemically related to thalidomide, but the significance of this relationship is unclear.

Some organophosphates have chemical structures that mimic male or female sex hormones, raising the concern that they may impact the fetus and the young infant during their early development. This has been clearly demonstrated to oc- cur in relatively uncomplicated organisms, but the same effect has not been shown in large animals or people. The relevance of this finding to health effects in humans is not yet clear.

Most regulatory agencies use the best data available, and then apply "safety factors" intended to compensate for these uncertainties. For example, the United States Environmental Protection Agency applies a 10-fold safety factor to compensate for differences between individuals, then another 10-fold safety factor to compensate for differences between species. Since late 2001, the U.S. EPA has been applying yet another 10-fold safety factor to compensate for differences between human infants and human adults. Despite these safety factors, good scientific data does not exist at this time to determine if the regulatory limits resulting from application of these safety factors provide too much or too little protection.

In summary, the safety data that is available is limited, with much of it coming from animal data. It is hard to tell exactly how this applies to people. Various experts have arrived at differing conclusions based on the same data.

\section{Q. Can I wash pesticides off of my foods before eating?}

A. Systemic pesticides are incorporated within the plant, and cannot be washed off. Non-systemic pesticides are on the surface of the plant. The amount of non-systemic pesticides on the surface of the fruit or vegetable can be reduced by washing the food with soap and water. As pediatricians, we don't recommend peeling when it is no longer a choking hazard since the peel contains dietary fiber and nutrients that are not necessarily found in the "meat" of the plant.

In summary, the extent to which pesticides can be washed off of foods varies depending on the chemical and the time lapse between the chemical application and the time of eating.

\section{Q. What about meats and poultry? Should I worry about them?}

A. Most commercially grown meat and poultry have been exposed to various chemicals during the raising period. It is difficult for the consumer to determine which chemicals have been used and also difficult to determine how much remain. The U.S. Food and Drug Administration has imposed standards to govern against the use of chemicals which pose the most risk in implementing the Food Quality Protection Act of 1996. Consumers who remain concerned may consider purchasing "Certified Organic" produce, poultry, and beef. However, it is unclear whether the additional cost of "Certified Organic" food reduces overall risk of harm from food.

When purchasing "organic" food, be aware that these foods may not be nutritionally fortified with recommended vitamins and minerals. Such vitamins and minerals include 
the addition of small amounts of iron to infant formula, folate to bread, iodine to salt, vitamin D to milk. This practice has been shown to reduce the frequency and severity of conditions such as iron deficiency causing anemia, folate causing congenital defects in newborn infants, iodine deficiency causing thyroid disease, and vitamin $\mathrm{D}$ reducing bone problems such as rickets.

\section{SUMMARY RECOMMENDATIONS:}

Pesticides and other agriculturally applied chemicals often are present in food and in people's bodies in small amounts.

Children are exposed to pesticides by varied routes, including through the food they eat, but the amount of exposure is rarely high enough to cause symptoms. However, some children, particularly the fetus and young infant, are at an increased risk from these chemicals.

The risks from the pesticides in foods are small compared to the tremendous health benefits of eating fruits and vegetables.

Agricultural chemicals are also found in animal products such as meat and dairy. Most of those chemicals are stored in the fat of the animal, so reducing the amount of animal fat in the diet will reduce your exposure to these chemicals as well as reduce other health risks associated with diets high in animal fat.

\section{SOURCES FOR ADDITIONAL READING}

Note: each of these publications has extensive references to buttress their points, and may be worth exploring for this reason if none other.

American Academy of Pediatrics Committee on Environmental Health. Etzel RA, Balk SJ, editors. Pediatric Environmental Health, 2nd Edition. Elk Grove Village, IL: American Academy of Pediatrics. 2003.

Brent RL, Tanski S, Weitzman M. A Pediatric Perspective on the Unique Vulnerability and Resilience of the Embryo and the Child to Environmental Toxicants: The Importance of Rigorous Research Concerning Age and Agent. Pediatrics 2004;113 (4 Supplement): 935-944.

Garry VF. Pesticides and Children. Toxicol Appl Pharmacol 2004; $198: 152-163$.

Kamel F, Hoppin JA. Association of Pesticide Exposure with Neurologic Dysfunction and Disease. Environ Health Perspect 2004; 112 (9): 950-958.

Reigart JR, Roberts JR. Pesticides in Children. Ped Clin N Am 2001;48 (5): 1185-98.

Weiss B, Amler S, Amler RW. Pesticides. Pediatrics 2004;113 (4 Supplement): 1030-1036. 